\title{
QUALITY OF MEDICAL LABORATORY SERVICES IN RESOURCE-LIMITED SETTINGS
}

\section{Bernardo Elias Correa Soares}

Department of Health Sanitation, Division of Biosafety, National School of Public Health, Fundação Oswaldo Cruz, NuBio /Fiocruz Av. Brasil 4036 sl 716, Rio de Janeiro, Brazil

Email: becorrea@ensp.fiocruz.br

\section{Summary}

This report endorses the author's own views on the subject after taking up a laboratory adviser mission in Africa. Taking the example of laboratory services practice in sub-Saharan countries, it is shown that diagnosis of diseases which require the use of laboratory suffer from lapses in the quality of case-detection and case-reporting. These services lack management and an information system that limits the set up of a laboratory network at national level. An efficient health program set in resource information system that limits the set up of a laboratory network at national leve
deprived countries would then yield improvements on systems and infrastructure.

\section{Overview}

The critical nature of emergency medical services in Saharan and Sub-Saharan Africa is paramount as development during the 21 st century is poised to arouse Africa to become a global economic player. Emergency care is akin to emergency medical response teams that can arrive on-site with a laboratory where blood testing, transfusion or mainly diagnosis of communicable diseases is essential. Economic constraints limit the development of such a network within the context of establishing facilities and health structures (Hébert et al., 2005). Poverty is the most quoted reason for the lack of these services as, according to Loefler (1998), there is "shortage of facilities, equipment, dressings and drugs, notably antibiotics." Supporting variables include overcrowding in populated areas where stand-alone facilities exist, insufficient environmental hygiene conditions conducive to the spread of disease, and insufficient maggot control leading to a large infestation of flies throughout large areas.

As an example, Onwujekwe et al. (2005) observed the relationship between the socio-economic status of a household and diagnosis and treatment in Nigeria. It was seen, as it is the case in most Sub-Saharan countries that poorest people seek care from 'low-level' providers, such as traditional healers and community health workers, due to their severe budget constraints while the least-poor group was more likely to rely on laboratory tests for diagnosis and to visit hospitals when seeking treatment. Improvements in the quality of diagnosis and treatment by the providers would help to redress this inequity, at least in the short- to medium-term.

In general, in poor countries many affected families can hardly afford even the cost of basic laboratory diagnostic tests, and some otherwise treatable conditions may lead to early death in affected patients. Most patients would be treated on the basis of clinical assessment only, since they could not afford the cost. Frustrated by this experience, they abscond or leave against medical . So availability of diagnosis facilities and necessary chemotherapeutic agents at affordable cost are vital for effective management of infections. Some studies like this of Onwujekwe et al. (2005) report that, in a few cases, although laboratory facilities were available, they were not accessible to all patients. Most patients could not afford the costs of confirmatory laboratory tests, which could cause the treatment to be started without pathological confirmation and would enhance the number of misdiagnosed cases to "adverse effects of agents with no clinical benefits at all". Also worth mentioning is the laboratory work as a critical point for providing on-site blood transfusions (Hébert et al., 2005). In developing countries, there are numerous diseases which require the use of blood transfusions and WHO (2001) reports that developing nations suffering from lapses in laboratory screening result in $31 \%$ of all transfusions while one-third are not screened at all for HIV, Hepatitis B or Hepatitis C.

\section{Case: Mozambique}

In the case of Mozambique, an African nation which perhaps can be understood as a case indicative of the environmental assessment one would find throughout the continent and therefore, can be labeled to be a median statistical nation. A nation representing the median would indicate that half of the nations that are categorized as resourced deficient, half would be above Mozambique standards in terms of resource allocation and half would fall below.

The quality of HIV/AIDS case-detection and case-reporting system in Mozambique was assayed by Chilundo, Sahay \& Sundby (2004), who propose the lack of management systems necessary to do the basic work of haematology, including medical laboratory procedures and analysis as a major constraint. The inherent flaws in the system are such that the testing performed on labound level, nay or may ot on results, whether positive or negative, then are targeted for misplacement or another form of mismanagement along the transmission of an information chain, where the lack of a strong information system prevents the results from the field from entering a reportable 
database at a central location. These evidences could be confirmed by own experience, during the author's medical mission as a laboratory adviser in the Mozambique hinterland in 2007.

Therefore, HIV/AIDS case-reporting is compromised by poor data quality, including under-reporting and discrepancies across different reporting channels and organizational levels, as well as a lack of standardized data forms and report formats. Similar to most African countries, the majority of testing facilities in Mozambique use the rapid or simple assays as both a screening and confirmatory test (WHO, 2001) ${ }^{\circ}$. As with most HIV testing, the simple assay is serum based test and therefore detects the presence of antibodies by the form of $t$-cell count instead of detecting the presence of the actual human immune-deficiency virus. It is termed a rapid test as the results are obtained in minutes and do not require the use of reagents or of any additional equipment. These tests can be easily conducted in a clinic (on-site testing) or in laboratories without electricity or those having limited infrastructure (i.e. lacking highly-skilled staff and special equipment). Thus, these assays are compatible with the existing constraints that exist nationwide in Mozambique's peripheral testing facilities. The commercial rapid tests currently used in all provinces and districts present sensitivity to operating and environmental temperatures. According to Chilundo, Sahay \& Sundby (2004) ${ }^{\circ}$ as well as the author's personal view, to operating and environmental temperatures. According to Chilundo, Sahay \& Sundby (2004) as well as the author's personal view,
HIV tests are routinely performed in hospitals, blood banks and clinical laboratories at the capital city or at a neighboring country, in this case using flow citometry techniques. Usually blood banks and laboratories use test kits with a shelf life of 18 months at temperatures of $28 / 30^{\circ} \mathrm{C}$, which is low for Mozambique, where heat may easily reach $38 / 40^{\circ} \mathrm{C}$ or more.

\section{Recommendations}

The issue of quality control where laboratory resources are lacking is of additional concern. Quality assessment of laboratory results is expected to be performed on a regular basis and although on-site evaluation through supervision visits by either provincial manager regularly occurred at hospital centers, the laboratories and blood banks appeared to be infrequently supervised. For example, manager regularly occurred at hospital centers, the laboratories and blood banks appeared to be infrequently supervised. For example,
a testing facility using outdated test kits had not been visited by a supervisor within two years; despite it was located only 30 kilometres a testing facility using outdated test kits had not been visited by a supervisor within two years; despite it was located only 30
from provincial headquarters.”

In a resource deprived environment, the first action to increase laboratory facilities is to obtain resources from an

organization. As the world develops through the unification of a global economy, a health network set up within resource deprived countries would yield improvements on current systems and enable new strong systems to replace the laboratory network, putting infrastructure into place.

\section{References}

1. Chilundo, B., Sundeep S., Sundby J. (2004). The Quality of HIV/AIDS case-detection and case reporting systems in Mozambique. African Journal of AIDS Research, 145-155.

2. Hébert PC, Wells G, Blajchman MA, Graham ID (2005). A multicenter, randomized, controlled clinical trial of transfusion requirements in critical care. Transfusion Requirements in Critical Care Medicine, 7-12.

3. Loefler, I. (1998). Surgical wound infection in the Third World: the African experience. Journal Medical Microbiology 47:471473.

4. Onwujekew, O., Ojukwu J., Uzochukwu, B., Dike, N., Ikeme, A., Shu, E. (2005). Where do people from different socioeconomic groups receive diagnosis and treatment for presumptive malaria, in south-eastern Nigeria ? Annals Tropical Medicine \& Parasitology 99(5):473-481.

5. WHO (2001). World Health Organization /UNAIDS - The global strategy framework on HIV/AIDS. WHO, Geneva ; 2001. 